\title{
ERRATUM
}

\section{Thioredoxin participates in a cell death pathway induced by interferon and retinoid combination}

Xinrong Ma, Sreenivasu Karra, Daniel J Lindner, Junbo Hu, Sekhar PM Reddy, Adi Kimchi, Junji Yodoi and Dhananjaya V Kalvakolanu

Correction to: Oncogene 2001; 20: $3703-3715$

In the above paper, the last author's middle initial was given incorrectly. It is correct as given above. 